\title{
AUTOMATED GENERATION OF TEST UNITS FOR DIAGNOSIS AND DEVELOPMENT OF COGNITIVE SKILLS
}

\author{
T. Angelov ${ }^{1} *$, V. Sivakova ${ }^{2}$, G. Totkov ${ }^{1}$ \\ ${ }^{1}$ Computer Science Department, University of Plovdiv "Paisii Hilendarski”, Plovdiv, Bulgaria \\ ${ }^{2}$ Faculty of Pedagogy, University of Plovdiv "Paisii Hilendarski", Plovdiv, Bulgaria
}

\begin{abstract}
The paper presents the architecture of a web-based system for automated generation of test units for diagnostics and development of cognitive skills. The system supports a database (DB) containing information objects of different type (text, images), including metadata used in the generation process. The database is updated through queries to external information sources (to supply the objects themselves) and with next addition of attributes. For example, an image can be accompanied by a textual description and metadata (as part of an e-learning process), a Bulgarian lexema - with automatically generated phonetic transcription and syllabicate, and so on. The supported and updated specific image metadata system (including color, size, purpose, etc.) and for lexams (composite sounds, syllables, etc.) provides an opportunity to generate test units for diagnosis and cognitive development. The selection of objects from DB suitable for insertion in a test unit of a certain type (for example, to distinguish objects with different characteristics) is performed by a query to the database for extraction of objects according to the value of the accompanying metadata.
\end{abstract}

Key words: cognitive skills, cognitive language assessment tasks, cognitive task model, automated test unit generation.

\section{INTRODUCTION}

Information and computer technologies (ICT) play an important role in the life of modern man and his education. The use of a computer in the education allows better organization of the education in the schools and the ways and methods of presenting the verbal and visual material.

The turbulent development of ICT creates conditions for the application of new methods and forms of education (especially of elearning), diagnostics and development of various cognitive skills, as well as mass distribution of didactic tests, online forms of quests and surveys, etc. The interest in the test as an element of education is also increasing with the massive use of computers and other electronic devices and the Internet and attempts to use it in the process of automating learning. The interest in the test increases with the tendency for the massive use of interactive boards in education.

*Correspondence to: Todor Angelov, University of Plovdiv "Paisii Hilendarski", Computer Science

Dept., 24 Tzar Assen, 4000 Plovdiv, Bulgaria,

email: angelov.todor@gmail.com

128
The term cognitive skills (cognition) refers to the mental processes associated with the acquisition of knowledge, including thinking, understanding, memorizing, judging and solving problems, etc. (1).

Basis cognitive processes are: perceptions, thinking, reasoning (searching for reasons for beliefs, conclusion and actions), interpretation, organization of ideas, memory (with three related sub-processes: fixation, retention, reproduction of information), learning, language (verbalization), discrimination (distinction of irritants), symbolic representation, portrayal, categorization, classification, apperception (the process of perception of objects related to past experience), attention, faith, intuition(irrational knowledge), deduction, decision making, preferences for something, problem solving and tasks. Sometimes, cognitive processes are used to manipulate mental content. Some of the cognitive processes can be artificially challenged (by machines - computers). There is also taxonomy of cognitive processes (remembering, understanding, analyzing, synthesizing, evaluating, integrating - uniting 
and linking knowledge, metacognition - the ability to see what, how, and how well you do things to get a result, etc.) $(2,3,4)$.

By "cognitive tasks" we will understand test units (TU), for developing a cognitive skill. Cognitive tasks are several types depending on the skill that is being diagnosed or developed.

An example of cognitive tasks (so called "phonetic tasks") are TUs using words / texts (possibly accompanied by images) and data for phoneme system of the relevant language (including phoneme classification, phonetic transcription rules, syllables, rhyming, etc.). When creating them, the selection of lexemes with the presence / absence of a certain letter, phonemes (from a given group), or combinations of consonants, in a certain position, of lemmas of predetermined length or containing a certain syllable, etc., is necessary. We will note that the latter also requires the need to accompany systems for generating 'phonetic' TUs (as well as other types of TUs related to the language) with corresponding lexical resources (machine dictionaries) and means for automated analysis and synthesis of texts $(5,6)$.

Tests for the development of cognitive skills are actively used in education of pre-school children as well as tests for diagnostic of individual stages of their development. At the end of pre-school preparation, tests are also applied to diagnose the child's readiness for school. Cognitive tests for early diagnosis of delayed development for preschool children and pupils are also used in education.

Creating cognitive tests for diagnosis and development is a difficult task. In addition, such tests contain TUs, which include images and texts at the same time, and are accompanied by specific requirements (compliance with the styling limitations of TUs included, use of a limited stock, etc.).

The following approaches are possible when compounding TUs (7):

- author's ('manual' creation by experts in a specific subject area);

- automated generation based on a semantic description of the studied subject area;

- using computer linguistics techniques retrieving data from text and / or Internet sources, including 'questionnaire systems' etc.

- automated generation based on previous experience in e-testing;

- choosing from a standard set of template questions (with next editing for the subject area), etc.
In the process of generating $\mathrm{TU}$ of different types for building a test bank, traditional methods such as (8):

- methods of computer linguistics generating factual questions, by text processing;

- positive response theory (IRT) - based on the probability that each learner will give a correct answer to a test item;

- bee algorithm - based on the IRT and the organization of bee life;

- semantic network technologies standardized knowledge presentation (ontologies) and content structured based on ontologies.

Another approach is related to the idea of accumulation - evaluation and accumulation of answers to open-ended questions with next generation of TU from other types (7).

The forms for generating the test variants are also different: simple static template, simple, unparametrized choice from the bank, generating by template for search from the database, parameterized choice from the database using metadata for the test variant type, keywords, section, etc.

Software systems can be used to create TUs and tests (examples of online systems for creating test are ClassMaker, Easy Test Maker, iSpring Quizmaker, Google Forms, HotPotatoes, Testmoz, ProProfs, QuizMaker, SurveyGizmo, SurveyMonkey, Wufoo, Zoomerang etc. (9). Each e-learning system (Moodle, ATutor, ILIAS, Sakai, Google Classroom, PeU 2.0, eLSe, OntoQue, etc.) also has capabilities for TU and test generating (7).

In order to diagnose or develop cognitive skills in different languages, a number of web sites and applications, including mobile devices, are also featured on the Internet: Luminosity: Brain games \& Brain Training (10), Brain Games, Brain Training \& Brain Exercises (11), NeuroNation (12), Elevate Brain Training (13), Mind Games (14), educational games in Bulgarian language for natural science, numbers and numbers, sounds, environmental knowledge, riddles, puzzles, kitchen (15), the educational software "Together at School" (in Bulgarian) with three modules - "Logic", "Mathematics" and "Reading" (16).

Despite the variety of software systems and Internet applications, many of the types of TU involved in the cognitive and cognitive development tests (especially for the development of phonetic and lexical knowledge) cannot be implemented. Some of 
them are not offering TU with graphical, audio and / or video elements, and tests created by one user cannot be used from another. Existing web-based imaging libraries are inappropriate for use when creating diagnostic tests and developing cognitive skills. On the other hand, the issue of automated generation of TU (and tests) to develop specific cognitive skills remains unsolved.

The main purpose of the research is to create web-based software system for automated generation of test units for diagnosis and development of cognitive skills.

In order to create a system for generating "cognitive" TUs we had to solve the following problems:

- designing a database with information objects of different types (text, images) with the possibility to update through queries to external information sources to import new objects;

- offering a system of metadata suitable for describing the objects stored in the database (for images - color, size, purpose, etc., for lexemes - phonemes, syllables, etc.);

- automated generating of metadata for information objects depending on their type (accompanying images with text description and metadata, and Bulgarian lexemes with phonetic transcription and syllables, etc.);

- generating tests for diagnosis and cognitive development based on DB requests for automated selection of objects suitable for inclusion in a TU of a given type (for example, generation of TU for distinguishing objects with different characteristics is done by request to the database for retrieving a set of information objects with specified values of the accompanying metadata).

The development of the system requires a classification of TU types used in the diagnosis and development of cognitive skills (including language skills). The model of cognitive TU (17) 'covers' the most common TUs of a similar nature. In the case of generating "phonetic tasks", it is also necessary to create and use algorithms for phonetic analysis of lexemes in the relevant language (algorithms for transcription, synthesis and rhyming in the Bulgarian language, see (9)).

\section{MATERIALS AND METHODS} ARCHITECTURE OF A SYSTEM FOR GENERATING 'COGNITIVE' TU

The paper presents the architecture of a webbased system for automated TU generation for diagnosis and development of cognitive skills. The system supports a database containing information objects of different types (text, images), accompanied by metadata used in the generation process.

For the purpose of development, a number of cognitive tests for children (2-7 years) were analyzed such as input tests for 1, 2, 3 and a preparatory group in the kindergarten (approved by the Ministry of Education and Science), a diagnosis test for the child's readiness for school, tests for the development of cognitive skills for the kindergarten and others. $(18,19)$.

The analysis of models of the used cognitive test (specifically of phonetic type) indicates that the necessary elements of a system for automated diagnostic tests and the development of cognitive skills are subsystems for: A. Image management; B. Management of linguistic resources; C. Metadata management; D. Generation (by defined criteria) of TU of certain types; E. Generation of tests; F. Communication and user interaction (including an Internet access portal and security subsystem). The Scheme of the architecture of the system is presented in Figure 1. We will note the basic functional requirements for subsystems A. - E., which are followed in the design and implementation of the system we called AGTUCS.

Subsystem A. (image management) supports the following features:

- possibility for adding information objects of the image type;

- opportunity for importing new objects through queries to external information sources;

- generation of characteristics of image-type objects.

Subsystem B. (linguistic resources

management) supports:

- options for adding text objects;

- manages the use and editing of a dictionary with words;

- modeling the phonetic system and using algorithms for phonetic transcription, collocation, rhymoforms for information objects;

Subsystem C. (metadata management)

supports features for:

- managing a system of metadata;

- automated metadata generation for information objects;

- editing metadata.

Subsystem D. (test unit generation) supports features for: 
ANGELOV T., et al.

- automated TU generation based on a type of cognitive skill;

- management of TUs;

- editing TUs;

- export TUs.

Subsystem E. (test generation):

- automated generation of tests from TUs;

- maintenance of ready-made TU templates for developing and diagnosing cognitive skills;
- supports DB with tests and editing tests;

- managing the resolution of already created tests.

Subsystem F. (user access management):

- Internet access of users to system functionalities and subsystems, incl. public and controlled access

- System and Information Security.

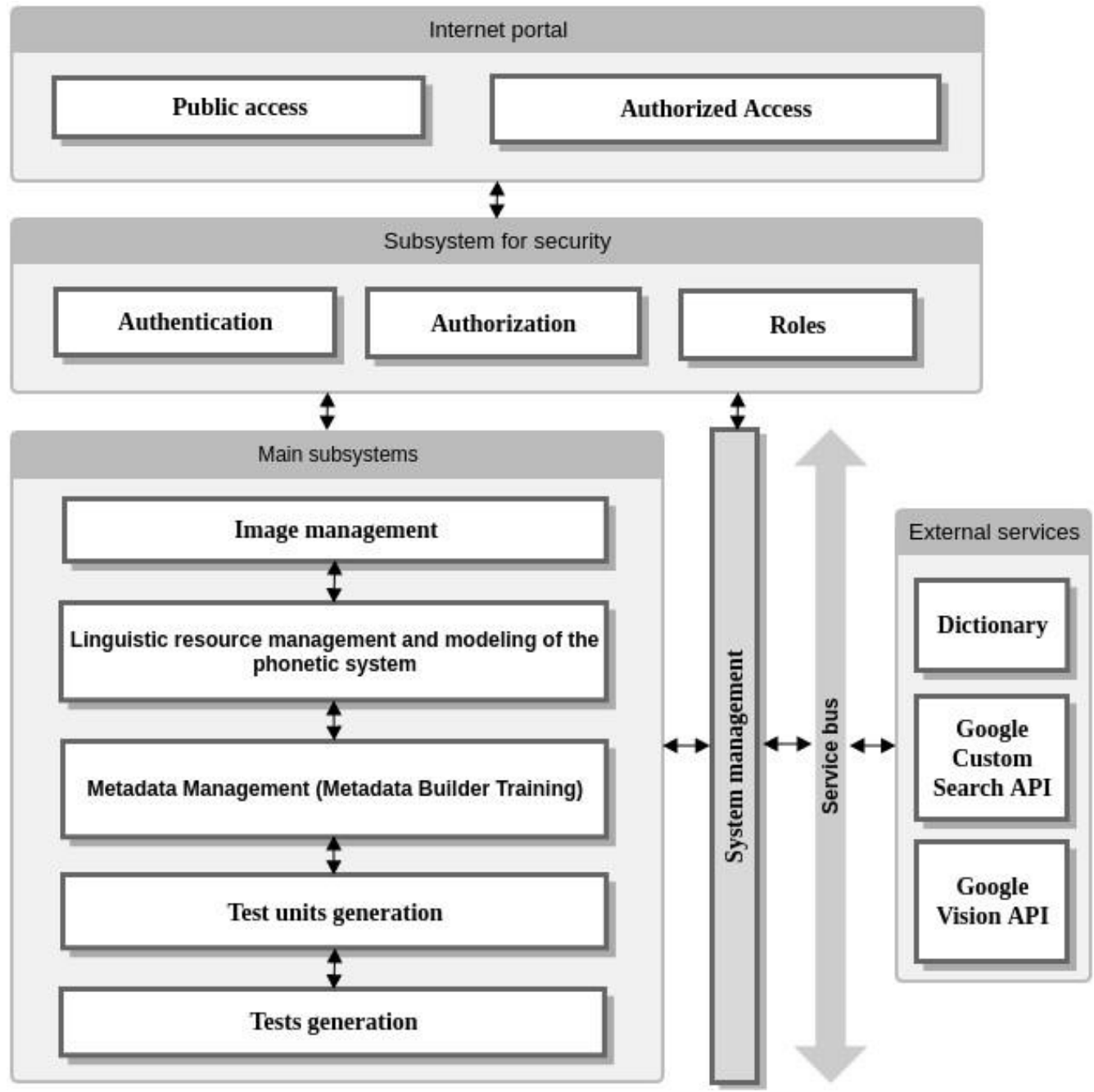

Figure 1. Architecture of a system for generating of test units for diagnosis and development of cognitive skills.

\section{SOFTWARE PROTOTYPES}

Implementation of the software prototype of the system uses micro-services based on RESTful API and Frontend JavaScript.

The RESTful API provides a reuse of some of the system's functionality when integrating with other systems and platforms (e.g. with Moodle), as well as building different user interfaces.

The software prototype is based on the architecture of Figure 1. and includes the realization of subsystems A. - F.

\section{Prototype of the subsystem A. Image management}

The functional capabilities of subsystem A imply using a relational database (DB). The prototype is built using MySQL to manage and store data.

Filling in the metadata for image is an automated process. We use the Google Vision API to connect to an external source.

Adding information objects of the image type can also be done manually in the system along with image metadata. 
We use queries to an external image module to add the image type information objects using the Google Custom Search API. It allows search customization, so the returned results to meet predefined criteria. In this case, it's customized to look for images on different sites. The found images are imported into the DB together with the metadata found for them. To optimize the system in terms of clientserver traffic, it is possible to dynamically or statically generate different sizes for the added images.

Prototype of the subsystem B. Management of linguistic resources and modeling of the phonetic system

A relational database is used for information objects of type "text". Adding objects can be done by using an external dictionary with words.

Algorithms for phonetic transcription, syllables and rhyming in BL are used. These algorithms are applied when adding information objects of a different type.

\section{Prototype of subsystem C. Managing metadata}

The metadata to the image objects are stored in the database. Hierarchical structure of metadata - a tree is maintained. Initially, certain metadata for objects such as form, material, sensitivity, etc. are introduced in the database and their characteristics, e.g. for shape - geometric shapes (square, rectangle, etc.). A loop of tasks is used to fill in the metadata values for existing metadata objects to an image. An image is randomly taken from the database with metadata and attributes and a question is asked, e.g.: "Is this circle?" or "Describe the form of the object". If the answer is positive then this feature is added to the specific image.

Adding new metadata that is not in the database is done manually in the system. As a specialist decides at which level (in the hierarchy) the new metadata will be added.

\section{Prototype subsystem D. Generation of test units}

The subsystem uses the DB for storage of TU. Based on the classification of the CTU types for a given cognitive skill, a set of CTUs for it are proposed.

The TU types that are implemented in the system are multiple choice, multiple response and short response, hot spot (select part of an image).
ANGELOV T., et al. The subsystem generates TU based on given input parameters. The subsystem maintains different TU templates relative to the type of cognitive skill that is being prepared.

The specialists can specify additional attributes like: duration, correct answer points, negative points for wrong answer, type, and more.

The TU may include information objects of different types (text, image).

The subsystem will support a feature that transfers ready-made TU from a accumulative type into Moodle. If TU is changed and sent to Moodle, it goes as a new TU. TU from an accumulative type are returned from Moodle with accumulated responses.

\section{Prototype subsystem E. Generation of tests}

Creating a test from TU is an automated process. Generating a test is based on DB queries for automated selection of objects suitable for inclusion in a TU of a given type (for example, generating a TU for distinguishing objects with different characteristics is performed by a query to the database to retrieve a set of information objects with specified metadata values).

\section{Prototype of subsystem F. for communication interaction (user access management)}

The subsystem includes user access control. There are two types of access: controlled and public. JWT (JSON Web Token) is used to ensure authentication and authorization, and its integration is multi-platform. On this principle, there is no problem for implementation of an Android app as well.

The authorization is implemented as a Role Base Access Control (RBAC).

Part of the functionality of the system is also available to unregistered users.

\section{RESULTS AND CONCLUSION}

We are conducting experiments with the system currently. An experimental study will be conducted with a group of teachers and other users of the system. The proposed system allows filling and editing text-type objects and images from different specialists. As well as creating test tasks and tests using objects from DB. The tests can be solved in the system. Test units can be exported to Moodle and used in tests.

With its help, cognitive test exercises, that are often used in practice and are oriented towards pre-school children, can be automatically generated. 


\section{REFERENCES}

1. What is Cognition? Meaning and Definition,

https://www.cognifit.com/cognition.

2. Radev, P., Main topics in psychology. University publishing, Plovdiv, 2014. ISBN 978-954-423-953-4. (in Bulgarian)

3. Sternberg, Robert J., Karin Sternberg. Cognitive psychology. Sixth Edition, 2012. ISBN -13 , ISBN -10 97811113447641111344760,609 p.

4. Sharpbrains - What are cognitive abilities and skills, and how to boost them, https://sharpbrains.com/blog/2006/12/18/ what-are-cognitive-abilities/

5. Totkov G., Methodology, Resources and Tools for Computerization of Bulgarian language (1988 - 2000), http://fmi.uniplovdiv.bg/GetResource?id=591

6. Sivakova V., G. Totkov. D. Blagoev. Phonetic Database for generating of logopedic exercises. ACM International Conference Proceeding Series; Proc. of the $11^{\text {th }}$ International Conference on Computer Systems and Technologies and Workshop for PhD Students in Computing 2011, Vienna, June 17-18, 2011, 558-563.

7. Totkov, G., M. Raykova, H. Kostadinova. Test in e-learning. First edition, Publisher Rakursi - Plovdiv, 2014, 217 p. ISBN: 978-954-8852-42-5. (in Bulgarian)

8. Automated test generation, Mariana Raykova http://old.nbu.bg/PUBLIC/IMAGES/File/ departments/informatics/Seminar_Marian a_Raykova.pdf (in Bulgarian)
ANGELOV T., et al.

9. Angelov, T. V. Sivakova, G. Totkov. Automated generation of phonetic and cognitive test units. Bulgarian language, literature and e-learning. P. 117-140. Publisher: Rakursi OOD, Plovdiv, 2014. ISBN 978-954-8852-47-0.

10. Luminosity, https://www.lumosity.com/

11. Fit brains - games for stimulating brain, http://www.fitbrains.com/

12. Neuronation, https://www.neuronation.com/

13. Elevate - brain training, https://www.elevateapp.com/

14. Mind http://mindgames.mindware.mobi/

15. Educational games, http://www.abc-bg.be

16. Educational software "Together at School, http://elasnas.bg/page/software

17. Angelov, T. V. Sivakova, G. Totkov. Automated Generating of Test Units for Evaluation of Cognitive and Language Skills. Proceedings of the National Conference on "Education and Research in the Information Society", Plovdiv, May, 2016, 079p-088p 2016. ISSN 13140752, http://hdl.handle.net/10525/2750

18. Batoeva, D., Pedagogical and psychological diagnostics. Study guide for children and students. Sofia: Askoni-izdat, 2007. ISBN 978-954-8542-89-0. (in Bulgarian)

19. Bijkov, G. and colleges., Test for diagnostic of readiness of children for school. Sofia: University publishing "St. Kliment Ohridski", Association "National testing center", 2011. 978-954-07-3208-4. (in Bulgarian) 